\title{
АСИМПТОТИЧЕСКИЕ СВОЙСТВА ДВУХШАГОВОГО ОПТИМАЛЬНОГО УПРАВЛЕНИЯ ЧАСТИЧНО НАБЛЮДАЕМЫМ СЛУЧАЙНЫМ ПРОЦЕССОМ
}

\author{
(Представил Н. Алумяэ)
}

Рассматривается частично наблюдаемый случайный процесс $(x, y)=\left(x_{t}, y_{t}: t=1,2\right)$, определяемый уравнениями

$$
x_{t}=u_{t} \beta_{t}^{1}+\beta_{t}^{0}, \quad y_{t}=x_{t}+h_{t},
$$

где $y$ - наблюдаемый, $x-$ ненаблюдаемый компонент процесса; $\beta=\left(\beta_{t}: t=1,2\right)-$ авторегрессионный (первого порядка) случайный процесс, $h=\left(h_{t}: t=1,2\right)$ - независимый, невырожденный гауссовый процесс. В $\left.{ }^{1}{ }^{1}\right]$ решена задача определения управлений $u_{1}^{*}, u_{2}^{*}\left(y_{1}\right)$, минимизирующих функционал

$$
J=M\left\{\left(x_{1}-x^{0}\right)^{2}+\left(x_{2}-x^{0}\right)\right\} .
$$

Здесь исследованы асимптотические свойства оптимального управления $u_{1}{ }^{*}$. при стремлении к бесконечности некоторых параметров задачи. Определены условия, когда оптимальное управление аппроксимируется сепарированным управлением. Полученные результаты предлагается использовать при исследовании многошаговых управляемых процессов $\left[{ }^{2}\right]$.

\section{1. Задача управления}

Пусть частично наблюдаемый управляемый случайный процесс $(\beta, x, y)=\left(\beta_{t}, x_{t}, y_{t}: t=1,2\right)$ задан уравнениями

$$
\begin{gathered}
\beta_{t}=\mu+\Phi\left(\beta_{t-1}-\mu\right)+\alpha_{t}, \quad \beta_{0}=\tilde{\beta}, \\
x_{t}=u_{t} \beta_{t}^{1}+\beta_{t}^{0} \\
y_{t}=x_{t}+h_{t}
\end{gathered}
$$

где $(\beta, x)$ - ненаблюдаемый, $y$ - наблюдаемый компонент процесса; $x_{t}$ - состояние, $u_{t}$ - управление, $\boldsymbol{\beta}_{t}=\left(\beta_{t}{ }^{0}, \beta_{t}{ }^{1}\right)^{\mathrm{T}}-$ вектор параметров управляемого объекта; $\mu$ - среднее, $\Phi$ - диагональная матрица параметров авторегрессии процесса $\beta$. Предполагается, что ошибки наблюдения $h_{t}, t=1,2$, импульсы обновления $\alpha_{t}, t=1,2$ и начальное условие $\tilde{\beta}=\left(\tilde{\beta}_{0}, \tilde{\beta}_{1}\right)^{\text {т }}$ уравнения (1) независимы попарно и во времени и распределены по невырожденному нормальному закону

$$
h_{t} \sim I N(0, r), \quad \alpha_{t} \sim I N(0, D), \quad \tilde{\beta} \sim N(\tilde{b}, \tilde{P}) .
$$

Ставится задача оптимального управления

$$
J=M\left\{\left(x_{1}-x^{0}\right)^{2}+\left(x_{2}-x^{0}\right)^{2}\right\} \rightarrow \inf _{u_{1}, u_{2}\left(u_{1}\right)},
$$


где $M$ - математическое ожидание, $x^{0}$ - эталонная величина. Класс допустимых управлений для $u_{2}\left(y_{1}\right)$ задан непрерывными функциями.

\section{2. Асимптотические свойства оптимального и сепарированного управления}

Задача управления (4) решена в [ $\left.{ }^{1}\right]$. Доказано, что оптимальное управление $u_{1}{ }^{*}$ на первом шаге (далее индекс времени $t=1$ не указывается) удовлетворяет условиям

$$
S_{u}+R_{u}=0, \quad S_{u u}+R_{u u} \geqslant 0,
$$

где $S_{u}, R_{u}$ и $S_{u u}, R_{u u}$ - первая и вторая производные от функций $S(u), R(u)$ соответственно. Согласно [ [ $\left.{ }^{1}\right]$,

$$
S=\left(a_{0}+u a_{1}-x^{0}\right)^{2}+G_{0}+u^{2} G_{1}
$$

если $T_{1}=0$, то

$$
R=P_{0}+\left[\left(c_{0}-x^{0}\right)^{2}+T_{0}^{2}\right] /\left(1+c_{1}^{\mathrm{T}} K_{1}^{-1} c_{1}\right),
$$

если $T_{1} \neq 0$, то

$R=P_{0}+\left(T_{0} / T_{1}\right)^{2}\left\{1-2 \sqrt{\pi}\left[L \operatorname{Im} w(z)+\left(Y-L^{2} / Y\right) \operatorname{Re} w(z) / 2\right]\right\} P_{1}$.

Здесь $a, c$ - оценки (прогнозы) параметров $\beta_{1}, \beta_{2}$, определяемые до момента поступления наблюдений по формулам

$$
a=\mu+\Phi(\widetilde{b}-\mu), \quad c=\mu+\Phi(a-\mu) ;
$$

$G, K$ - ковариации оценок $a, c$

$$
G=D+\Phi \widetilde{P} \Phi, \quad K=D+\Phi G \Phi
$$

функции $T, P, Y, L$, w определены следующим образом:

$$
\begin{gathered}
T=\Phi G v / \sqrt{r+v^{\mathrm{T}} G v}, \quad v^{\mathrm{T}}=(1, u) ; \\
P=K-T T^{\mathrm{T}} ; \quad 2 Y^{2}=P_{1} / T_{1}^{2} ; \\
\sqrt{2} L=\left(c_{0}-x^{0}\right) / T_{0}+\sqrt{2} X, \quad \sqrt{2} X=-c_{1} / T_{1} ; \\
w(z)=\mathrm{e}^{-z^{2}}\left(1+2(\pi)^{-1 / 2} i \int_{0}^{z} \mathrm{e}^{t^{2}} d t\right), \quad z=X+i Y .
\end{gathered}
$$

Индексами 0 и 1 обозначены соответственно первая и вторая координаты рассматриваемого вектора: $a, c, T, \mu$ (или диагональный элемент матрицы $G, P, K, \Phi, D)$.

Если случайный процесс $\beta^{1}$ детерминирован или процесс $\beta$ независим во времени, то задачи управления и прогноза возмущений $\beta_{t}{ }^{0}$ или $\beta_{t}$, $t=1,2$ разделяются, так как $R_{u}=0$. Поэтому оптимальным оказывается сепарированное управление

$$
u^{0}=-a_{1}\left(a_{0}-x^{0}\right) /\left(a_{1}^{2}+G_{1}\right),
$$

определяемое из условий $S_{u}=0, S_{u u}>0$. В общем случае $R_{u} \neq 0$, в связи с чем возникает вопрос: при каких условиях задача оптимального управления разделяется асимптотически в том смысле, что существует малый параметр $\varepsilon>0$ такой, что $u_{\varepsilon}{ }^{*} \rightarrow b$ и $u_{\varepsilon}{ }^{0} \rightarrow b$ при $\varepsilon \rightarrow 0$ ? Здесь $b$ - некоторая константа. В случае неразделяемости интересуемся асимптотическим поведением оптимального и сепарированного управления. Чтобы найти ответы на эти вопросы, определим скорости роста 
функций $S_{u}, R_{u}$ при увеличении одного или нескольких параметров из множества $\left\{x^{0}, \Phi_{i}, D_{i}, \widetilde{b}_{i}, \widetilde{P}_{i}: i=1,2\right\}$, задаваемого постановкой задачи. Воспользуемся стандартным обозначением $\delta_{n}=O\left(\varrho_{n}\right)$, где $\left\{\delta_{n}: n=\right.$ $=1,2, \ldots\}$ - последовательность реальных чисел, $\left\{\varrho_{n}: n=1,2, \ldots\right\}$ монотонно возрастающая последовательность положительных чисел такая, что соотношение $\delta_{n} / \varrho_{n}$ ограничено. В Приложении показано, что при любом ограниченном управлении

$$
S_{u}\left(u, \varrho_{n}\right)=O\left(\varrho_{n}^{p}\right),
$$

причем

1) если $D_{0, n}=O\left(\mathrm{on}^{2}\right)$, то $p=0$;

2) если $\left|x_{n}{ }^{0}\right|=O\left(\varrho_{n}\right)$ или $\left|\Phi_{0, n}\right|=O\left(\varrho_{n}\right)$, или $\left|\widetilde{b}_{0, n}\right|=O\left(\varrho_{n}\right)$, $\widetilde{P}_{0, n}=O\left(\mathrm{Q}^{2}\right)$, то $p=1$;

3) если $\left|\Phi_{1, n}\right|=O\left(\varrho_{n}\right)$ или $D_{1, n}=O\left(\varrho_{n}^{2}\right)$, или $D_{i, n}=O\left(\varrho_{n}^{2}\right), i=0,1$, или $\left|\widetilde{b}_{1, n}\right|=O\left(\varrho_{n}\right), \widetilde{P}_{1, n}=O\left(\varrho_{n}^{2}\right)$, или $\left|\widetilde{b}_{i, n}\right|=O\left(\varrho_{n}\right), \widetilde{P}_{i, n}=O\left(\varrho^{2}\right)$, $i=0,1$, то $p=2$.

Там же показано, что

$$
R_{u}\left(u, \varrho_{n}\right)=O\left(\varrho_{n}^{q}\right)
$$

и доказаны леммы 1 и 2.

Л ем а 1. 1) Если $\left|\Phi_{1, n}\right|=O\left(\varrho_{n}\right)$ или $\left|\widetilde{b}_{1, n}\right|=O\left(\varrho_{n}\right), \widetilde{P}_{1, n}=O\left(\varrho_{n}^{2}\right)$, то $q=-1$.

2) Если $\left|x_{n}{ }^{0}\right|=O\left(\varrho_{n}\right)$ или $\left|\widetilde{b}_{i, n}\right|=O(\varrho n), \widetilde{P}_{i, n}=O\left(\varrho n^{2}\right), i=0,1$, то $q=2$.

3) Пусть $Y>3$. Если $D_{1, n}=O\left(\varrho n^{2}\right)$, то $q=-2$. Если $D_{i, n}=O\left(\varrho n^{2}\right)$, $i=0,1$, то $q=2$.

4) Пусть $\left|X_{n}\right|>3,9$ или $Y_{n}>3$ для каждого $n=1,2, \ldots$. Если $D_{0, n}=O\left(\varrho_{n}^{2}\right)$ uли $\left|\widetilde{b}_{0, n}\right|=O\left(\varrho_{n}\right), \quad \widetilde{P}_{0, n}=O\left(\varrho_{n}^{2}\right)$, то $q=6$. Eсли $\left|\Phi_{0, n}\right|=O\left(\varrho_{n}\right)$, то $q=8$.

Л е м а 2. Пусть последовательность возмущзений $\beta^{0}$ независима. Тогда

1) если $\left|\Phi_{1, n}\right|=O\left(\varrho_{n}\right)$ или $\left|\widetilde{b}_{1, n}\right|=O\left(\varrho_{n}\right), \widetilde{P}_{1, n}=O\left(\varrho_{n}^{2}\right)$ uли $\left|\widetilde{b}_{i, n}\right|=$ $=O\left(\varrho_{n}\right), \widetilde{P}_{i, n}=O\left(\varrho^{2}\right), i=0,1$, то $q=-2$;

2) пусть $Y>3$. Если $D_{i, n}=O\left(\mathrm{\varrho n}^{2}\right), i=0,1$, то $q=0$;

3) пусть $\left|X_{n}\right|>3,9$ или $Y_{n}>3$ для каждого $n=1,2, \ldots$. Если $D_{0, n}=O\left(\varrho_{n}^{2}\right)$, то $q=2$.

В силу (9) и (10) при всех $\varrho>0$ выполняется

$$
S_{u}(u, \varrho)=\varrho^{p} \widetilde{S}_{u}(u, \varrho), \quad R_{u}(u, \varrho)=\varrho^{q} \widetilde{R}_{u}(u, \varrho),
$$

где $\widetilde{S}_{u}(u, \cdot), \widetilde{R}_{u}(\cdot, \cdot)$ - ограниченные функции по е и $(u, \varrho)$ соответственно. Таким образом, оптимальное управление $u_{\varepsilon}{ }^{*}$ и сепарированное управление $u_{\varepsilon}{ }^{0}$ определяются соответственно из условий

$$
\begin{array}{ll}
\widetilde{S}_{u}\left(u_{\varepsilon}, \varrho\right)+\varepsilon \widetilde{R}_{u}\left(u_{\varepsilon}, \varrho\right)=0, & \widetilde{S}_{u u}+\varepsilon \widetilde{R}_{u u}>0 ; \\
\widetilde{S}_{u}^{\prime}\left(u_{\varepsilon}, \varrho\right)=0, & \widetilde{S}_{u u}>0,
\end{array}
$$

где $\varepsilon=Q^{q-p} ; \widetilde{S}_{u u}, \widetilde{R}_{u u}-$ производные от функций $\widetilde{S}_{u}, \widetilde{R}_{u}$. В силу лемм 1 и 2 условие $p>q$ выполняется, если к бесконечности стремятся или параметры $\widetilde{b}_{1}, \widetilde{P}_{1}$, или параметр $\Phi_{1}$, или $D_{1}$. Данное условие выполняется и в том случае, если процесс возмущений $\beta^{0}$ независим и параметры $D_{i}$ или $\widetilde{b}_{i}, \widetilde{P}_{i}, i=0,1$ стремятся к бесконечности.

Докажем утверждение. 
Утвер ждение 1. Пусть $\varepsilon>0 u \varepsilon \rightarrow 0$. Если выполняются условия, при которых $p>q$, то $u_{\varepsilon}{ }^{0} \rightarrow 0 u u_{\varepsilon}{ }^{*} \rightarrow 0$.

Д ок а з а тел ь ст в о. Согласно [ $\left.{ }^{1}\right]$, в принятых условиях как оптимальное управление $u_{\varepsilon}{ }^{*}$, так и сепарированное управление $u_{\varepsilon}{ }^{0}$ существует при всех $\varepsilon>0$. Пусть $\left\{\delta_{n}\right\}$ произвольная, сходящаяся к нулю последовательность. Рассмотрим уравнение

$$
\widetilde{S}_{u}\left(u_{\varepsilon}, \varrho\right)=\delta_{n}
$$

и обозначим решение этого уравнения через $u_{\varepsilon}\left(\delta_{n}\right)$. Очевидно, что

$$
\lim _{n \rightarrow \infty} u_{\varepsilon}\left(\delta_{n}\right)=u_{\varepsilon}^{0}=0 \text {. }
$$

Так как $\widetilde{R}_{u}\left(u_{\varepsilon}, \varrho\right)$ - ограниченная функция, то отсюда вытекает, что $u_{\varepsilon}{ }^{*} \rightarrow 0$ при $\varepsilon \rightarrow 0$. Утверждение доказано.

Перечислим условия (согласно леммам 1 и 2 , они соответствуют случаю $p<q)$.

(А.1) $\mathrm{K}$ бесконечности стремится или параметр $x^{0}$, или параметры $\tilde{b}_{0}$, $\widetilde{P}_{0}$, или

(А.2) параметр $\Phi_{0}$, или

(А.3) один параметр из множества $\left\{x^{0}, \Phi_{0}, \widetilde{b}_{0}\right\}$.

Очевидно, что

$$
R(u, \varrho)=\varrho^{m} \widetilde{R}(u, \varrho),
$$

где $\widetilde{R}(u, \varrho)$ - ограниченная функция. В условиях (А.1), (А.2) $m>0$. Перечислим гипотезы.

(Г.1) Функция $\widetilde{R}(u, \varrho)$ строго возрастает на интервале $(-\infty, l(\varrho))$ и строго убывает на интервале $(l(\varrho), \infty)$ для каждого $\varrho>\varrho$.

$(Г .2)$ Функция $\widetilde{R}(u, \varrho)$ строго убывает на интервале $(-\infty, 0)$ и строго возрастает на интервале $(0, \infty)$ для каждого @ $>$ Q.

Здесь $\varrho_{i}$ - положительная константа, $l(\mathrm{~g})$ - ограниченная функция. В случае выполнения условия (А.1) или (А.2) гипотезы (Г.1) или (Г.2) соответственно подтверждаются численным экспериментом. Докажем утверждение.

Утвер ждение 2. Пусть $1 / \varepsilon>0 u \varepsilon \rightarrow \infty$. Тогда

1) если выполняются условия (А.1) и (Г.1), то $\left|u_{\varepsilon}{ }^{*}\right| \rightarrow \infty$, а если (А.2) $u\left(\right.$ Г.2) $-u_{\varepsilon}^{*} \rightarrow 0$;

2) если выполняется условие (A.3), то $\left|u_{\varepsilon}{ }^{0}\right| \rightarrow \infty$.

Доказательство. Если выполняется условие (А.1), то согласно $(\Gamma .1), \widetilde{R}(u, \varrho) \rightarrow \min$ при $u \rightarrow \infty$ или $u \rightarrow-\infty$. Так как функция $\widetilde{S}_{u}\left(u_{\varepsilon}, \varrho\right)$ ограничена в силу решаемости задачи (11) и ограниченности предела

$$
\lim _{\varepsilon \rightarrow \infty} \tilde{S}_{u}\left(u_{\varepsilon}, \varrho\right)=B
$$

то из (11) можно видеть, что $\left|u_{\varepsilon}^{*}\right| \rightarrow \infty$.

Если выполняется условие (А.2), то согласно $(\Gamma .2), \widetilde{R}(u, \varrho) \rightarrow \min$ при $u \rightarrow 0$. Так как функция $\widetilde{S}_{u}\left(u_{\varepsilon}, \varrho\right)$ ограничена, то отсюда вытекает, что $u_{\varepsilon}{ }^{*} \rightarrow 0$.

Если выполняется условие (А.3), то $\left|u_{\varepsilon}{ }^{0}\right| \rightarrow \infty$, что доказывается аналогично утверждению 1 при помощи стремящейся к бесконечности последовательности $\left\{\delta_{n}\right\}$. 


\section{3. Обсуждение результатов}

Интерпретируем полученные выше результаты в трех случаях:

1. Пусть $p>q$. Тогда по мере уменьшения параметра $\varepsilon$ как оптимальное, так и сепарированное управление стремятся к нулю. Поэтому, если $\varepsilon$ достаточно мал, то оптимальное управление аппроксимируется сепарированным управлением. Это соблюдается в том случае, если случайный процесс $\beta^{1}$ существенно нестационарный, т. е. $\left|\Phi_{1}\right|>1$, или существенно недетерминированный (непредсказуемый), т. е. величина дисперсии $D_{1}$ достаточно большая, или если $M\left\{\tilde{\beta}_{1}^{2}\right\}$ - неопределенность начальных условий процесса $\beta^{1}$ - достаточно большая. А также в том случае, если процесс возмущений $\beta^{0}$ независим и дисперсии $D_{i}, i=0,1$ или $M\left\{\tilde{\beta^{2}}\right\}-$ неопределенность начальных условий случайного процесса $\beta$ - достаточно большие.

2. Пусть $p<q$. Тогда возможны два случая:

a) по мере роста параметра $\varepsilon$ как оптимальное, так и сепарированное управление стремятся к бесконечности или

б) оптимальное управление стремится к нулю, а сепарированное - к бесконечности.

Таким образом, если нашей целью является максимизация (минимизация) ненаблюдаемого выходного процесса $x$ или если $M\left\{\tilde{\beta}_{0}^{2}\right\}-$ неопределенность начальных условий процесса $\beta^{0}-$ большая, то оптимальное управление аппроксимируется сепарированным управлением и не аппроксимируется, если процесс возмущений $\beta^{0}$ существенно нестационарный.

3. Пусть $p=q$. Тогда задача управления не имеет малого (большого) параметра $\varepsilon=1$; точность аппроксимации оптимального управления сепарированным не зависит от условий задачи, при которых $p=q$. Таким, например, являются влияние параметров $\widetilde{b}, \widetilde{P}$ и, если процесс возмущений $\beta^{0}$ зависим, влияние ковариаций $D_{i}, i=0,1$ процесса обновления.

Авторы выражают благодарность Т. Тобиасу за полезные замечания.

\section{ПРИЛОЖЕНИЕ}

\section{Доказательство лемм 1 и 2}

\section{1. Доказываем следующие утверждения:}

Лемм а 3. Пусть $Y>3$. Если $\left|X_{n}\right|=O\left(\varrho_{n}^{-1}\right)$, то $\operatorname{Re} w\left(z_{n}\right)=O(1)$, $\operatorname{Im} w\left(z_{n}\right)=O\left(\mathrm{en}^{-1}\right)$. Пусть $\left|X_{n}\right|>3,9$ или $Y_{n}>3$ для каждого $n=1,2, \ldots$. Ecлu $\left|X_{n}\right|=O\left(\varrho_{n}\right)$ u $Y_{n}=O(\varrho n)$, то Re $w\left(z_{n}\right)=O\left(\varrho_{n}^{-1}\right)$, $\operatorname{Im} w\left(z_{n}\right)=O\left(\varrho^{-1}\right)$.

Доказ ательство. Пусть $|X|>3,9$ или $Y>3$. Тогда, согласно $\left[{ }^{3}\right]$, в этой области функция $(z)$ ограничена и аппроксимируется с большой точностью $\left(|\varepsilon|<2 \cdot 10^{-6}\right)$ при помощи полинома Эрмита третьего порядка

$$
w(z)=i z \sum_{j=1}^{3} H_{j} /\left(z^{2}-w_{j}\right)+\varepsilon,
$$

где $\varpi_{j}, H_{j}-$ нули и весовые множители полинома.

Используя (П.1), убеждаемся в том, что

$$
\operatorname{Re} w(z) \approx \sum_{j=1}^{3} H_{j}\left(E X-F_{j} Y\right) /\left(E^{2}+F_{j}^{2}\right)
$$




$$
\operatorname{Im} w(z) \approx \sum_{j=1}^{3} H_{j}\left(F_{j} X+E Y\right) /\left(E^{2}+F_{j}^{2}\right),
$$

где $F_{j}=X^{2}-Y^{2}-w_{j}, E=2 X Y$.

Если $Y>3$ и $\left|X_{n}\right|=O\left(\varrho_{n}^{-1}\right)$, то $F_{j}=O(1), E=O\left(\varrho n^{-1}\right)$. Поэтому

$$
\operatorname{Re} w\left(z_{n}\right)=O(1) ; \quad \operatorname{Im} w\left(z_{n}\right)=O\left(\varrho_{n}^{-1}\right) .
$$

Второе утверждение леммы доказывается аналогичным образом.

2. Дифференцируем по $u$ функцию (6)

$$
0,5 S_{u}=\left(a_{0}+u a_{1}-x^{0}\right) a_{1}+u G_{1}
$$

и функцию (8): После несложных, но громоздких преобразований получим

$$
0,5 R_{u}=\Lambda_{1}+2 \sqrt{\pi}\left(\Lambda_{2} \operatorname{Im} w(z)+\Lambda_{3} \operatorname{Re} w(z)\right),
$$

где $\Lambda_{i}=\lambda_{i} T_{0}^{2} / u$. Коэффициенты $\lambda_{i}$ определены следующим образом:

$$
\begin{aligned}
& \lambda_{1}=1-2 Y^{2}-Q\left[2 L X\left(1-2 Y^{2}\right)+\left(Y^{2}-L^{2}\right)\left(2 Y^{2}+1\right)\right], \\
& \lambda_{2}=Y^{2}(L+X)-Q\left\{0,5 X\left(4 Y^{2}+1\right)\left(L^{2}-Y^{2}\right)+L\left[2 Y^{2}\left(X^{2}-Y^{2}-1\right)-1\right]\right\}, \\
& \lambda_{3}=Y\left\{Y^{2}-L X-Q\left[\left(Y^{2}-L^{2}\right)\left(X^{2}-Y^{2}-1-0,25 / Y^{2}\right)+\right.\right. \\
& \left.\left.\quad+L\left(X\left(4 Y^{2}+1\right)-1\right)\right]\right\} .
\end{aligned}
$$

\begin{tabular}{|c|c|c|c|c|c|c|c|c|c|c|c|c|c|c|}
\hline Условия & $a_{i, n}$ & $G_{i, n}$ & $S_{u, n}$ & $T_{i, n}$ & $P_{1, n}$ & $X_{n}$ & $Y_{n}$ & $Q_{n} \mid$ & $\begin{array}{l}R_{u, n} \\
\Phi_{0}=0\end{array}$ & $L_{n}$ & $\Lambda_{1, n}$ & $\Lambda_{2, n}$ & $\Lambda_{3, n}$ & $R_{u, n}$ \\
\hline$\left|\Phi_{0, n}\right|=O\left(\varrho_{n}\right)$ & $\begin{array}{l}1 \\
0\end{array}$ & $\begin{array}{l}2 \\
0\end{array}$ & 1 & $\begin{array}{r}2 \\
-1\end{array}$ & 0 & 1 & 1 & 0 & - & 1 & 8 & 9 & 9 & 8 \\
\hline$\left|\Phi_{1, n}\right|=O\left(\varrho_{n}\right)$ & $\begin{array}{l}0 \\
1\end{array}$ & $\begin{array}{l}0 \\
2\end{array}$ & 2 & $\begin{array}{r}-1 \\
2\end{array}$ & 4 & 0 & 0 & -2 & -2 & 1 & -2 & -1 & -1 & -1 \\
\hline$D_{0, n}=O\left(\varrho_{n}^{2}\right)$ & $\begin{array}{l}0 \\
0\end{array}$ & $\begin{array}{l}2 \\
0\end{array}$ & 0 & $\begin{array}{r}1 \\
-1\end{array}$ & 0 & 1 & 1 & 0 & 2 & 1 & 6 & 7 & 7 & 6 \\
\hline$D_{1, n}=O\left(\mathrm{Q}_{n}^{2}\right)$ & $\begin{array}{l}0 \\
0\end{array}$ & $\begin{array}{l}0 \\
2\end{array}$ & 2 & $\begin{array}{r}-1 \\
1\end{array}$ & 2 & -1 & 0 & -2 & -2 & 1 & -2 & -1 & -2 & -2 \\
\hline $\begin{array}{l}D_{i, n}=O\left(\varrho_{n}^{2}\right) \\
i=0,1\end{array}$ & $\begin{array}{l}0 \\
0\end{array}$ & $\begin{array}{l}2 \\
2\end{array}$ & 2 & $\begin{array}{l}1 \\
1\end{array}$ & 2 & -1 & 0 & 0 & 0 & -1 & 2 & 1 & 2 & 2 \\
\hline $\begin{array}{l}\left|\widetilde{b}_{0, n}\right|=O\left(\varrho_{n}\right) \\
\widetilde{P}_{0, n}=O\left(\varrho_{n}^{2}\right)\end{array}$ & $\begin{array}{l}1 \\
0\end{array}$ & $\begin{array}{l}2 \\
0\end{array}$ & 1 & $\begin{array}{r}1 \\
-1\end{array}$ & 0 & 1 & 1 & 0 & - & 1 & 6 & 7 & 7 & 6 \\
\hline $\begin{array}{l}\left|\widetilde{b}_{1, n}\right|=O\left(\varrho_{n}\right), \\
\widetilde{P}_{1, n}=O\left(\varrho_{n}^{2}\right)\end{array}$ & $\begin{array}{l}0 \\
1\end{array}$ & $\begin{array}{l}0 \\
2\end{array}$ & 2 & $\begin{array}{r}-1 \\
1\end{array}$ & 2 . & 0 & 0 & -2 & -2 & 1 & -2 & -1 & -1 & -1 \\
\hline $\begin{array}{l}\left|\widetilde{\delta}_{i, n}\right|=O\left(\varrho_{n}\right), \\
\tilde{P}_{i, n}=O\left(\varrho_{n}^{2}\right), \\
i=0,1\end{array}$ & $\begin{array}{l}1 \\
1\end{array}$ & $\begin{array}{l}2 \\
2\end{array}$ & 2 & $\begin{array}{l}1 \\
1\end{array}$ & 2 & 0 & 0 & 0 & -2 & 0 & 2 & 2 & 2 & 2 \\
\hline$\left|x_{n}^{0}\right|=O\left(\varrho_{n}\right)$ & $\begin{array}{l}0 \\
0\end{array}$ & $\begin{array}{l}0 \\
0\end{array}$ & 1 & $\begin{array}{l}0 \\
0\end{array}$ & 0 & 0 & 0 & 0 & 2 & 1 & 2 & 2 & 2 & 2 \\
\hline
\end{tabular}

Здесь $Q=\left(r+G_{0}\right) /\left(r+G_{0}+u^{2} G_{1}\right)$.

Если $\Phi_{0}=0$, то функция (8) упрощается. Поэтому

$$
\begin{aligned}
R_{u}= & \left(\mu_{0}-x^{0}\right)^{2} Q\left\{2 Y^{2}+1-\sqrt{\pi}\left[X\left(4 Y^{2}+1\right) \operatorname{Im} w(z)+\right.\right. \\
& \left.\left.+\left(2 Y\left(Y^{2}-X^{2}+1\right)+0,5 / Y\right) \operatorname{Re} w(z)\right]\right\} / u .
\end{aligned}
$$


3. При помощи выражений (П.2)-(П.4) и утверждений леммы 3 можно показать, что при любом ограниченном управлении

$$
S_{u}\left(u, \varrho_{n}\right)=O\left(\varrho_{n}^{p}\right)
$$

и что при ненулевых управлениях

$$
R_{u}\left(u, \varrho_{n}\right)=O\left(\varrho_{n}^{q}\right),
$$

где $p$ и $q$ в зависимости от изменяемых параметров выражены по таблице.

Если $u=0$, то $T_{1}=0$ и, согласно $(7), R_{u}\left(0, \varrho_{n}\right)=0$.

\section{ЛИТ Е РАТ У РА}

1. Тенно Р. Изв. АН ЭССР, Физ. Матем., 32, № 1, 11-17 (1983).

2. Methods and applications in adaptive control. Proc. Intern. Symp. Bochum, 1980 (ed. by H. Unbehanen). Springer-Verlag, Berlin-Heidelberg-New York, 1980. 3. Справочник по спещиальным функциям (под ред. М. Абрамовича, И. Стигана). М., «Наука», 1979.

Ннститут кибернетики Академии наук Эстонской ССР
Поступила в редакцию 30/XII 1982

R. TENNO, H. OIT

\section{OSALISELT VAADELDAVA JUHUSLIKU PROTSESSI OPTIMAALJUHTIMISOLESANDE LAHENDI OMADUSI}

On uuritud ühe mittesepareeruva juhtimisülesande lahendi asümptootilisi omadusi, kui ülesande mõningad parameetrid lähenevad lôpmatusele, ning leitud tingimused, mille puhul separeeritud juhtimine aproksimeerib optimaalset.

\section{R. TENNO, H. OIT}

\section{LARGE PARAMETER PROPERTIES OF TWO-STEP OPTIMAL CONTROL OF THE PARTIALLY OBSERVABLE STOCHASTIC PROCESS}

It is assumed that a partially observable stochastic process $\left(\beta_{t}, x_{t}, y_{t}: t=1,2\right)$ is described by the equations (1)-(3), where $\left(y_{t}\right)$ is an observable process; $\left(\beta_{t}\right),\left(x_{t}\right)$ are an unobservable process; $\alpha_{t}$ and $h_{t}$ are independent nonsingular Gaussian variables. Let $\left(u_{1}^{*}, u_{2}{ }^{*}\left(y_{1}\right)\right)$ be a sequence of optimal controls which minimize a functional (4), and let $u_{1}{ }^{0}, u_{2}^{0}\left(y_{1}\right)$ be (separated) controls which minimize the functions

$$
J_{1}=M\left(x_{1}-x^{0}\right)^{2}, \quad \cdot J_{2}=M\left(x_{2}-x^{0}\right)^{2},
$$

respectively. It is known that $u_{2}{ }^{*}\left(y_{1}\right)=u_{2}{ }^{0}\left(y_{1}\right)$. For the first step we have shown the following large parameter properties of the optimal control $u_{1}^{*}$ and separated control $u_{1}^{0}$ : Let $1 / \mathrm{Q}>0$ and $\varrho \rightarrow \infty$.

a) If $M \tilde{\beta}_{1}=\varrho, \operatorname{cov} \tilde{\beta}_{1}=\varrho^{2}$ or $\Phi_{1}=\varrho$ or $D_{1}=\varrho^{2}$, then $u_{1}^{*}(\varrho) \rightarrow 0$ and $u_{1}{ }^{*}(\varrho) \rightarrow 0$.

b) If $x^{0}=\varrho$ or $M \tilde{\beta}_{0}=\varrho$, cov $\tilde{\beta}_{0}=\varrho^{2}$, then $\left|u_{1}{ }^{*}(\varrho)\right| \rightarrow \infty$ and $\left|u_{1}{ }^{0}(\varrho)\right| \rightarrow \infty$.

c) If $\Phi_{0}=\varrho$, then $u_{1}^{*}(\varrho) \rightarrow 0$ and $\left|u_{1}{ }^{\circ}(\varrho)\right| \rightarrow \infty$. 